\title{
Algal Bloom in Aquatic Ecosystems - An Overview
}

\section{MOZHGAN GHORBANI ${ }^{1}$, SEYED AHMAD MIRBAGHERI ${ }^{2 *}$, AMIR HESSAM HASSANI ${ }^{3}$, JAFAR NOURI ${ }^{4}$ and SEYED MASOUD MONAVARI ${ }^{5}$}

\author{
${ }^{1}$ Faculty of the Environment and Energy, Science and Research Branch, \\ Islamic Azad University, Tehran, Iran. \\ ${ }^{2 \star}$ Department of Environmental Engineering, Faculty of Environment and Energy, \\ Science and Research Branch, Islamic Azad University, Tehran, Iran. \\ ${ }^{3}$ Department of Environmental Engineering, Faculty of Environment and Energy, \\ Science and Research Branch, Islamic Azad University, Tehran, Iran. \\ ${ }^{4}$ Department of Environmental Management, Faculty of Environment and Energy, \\ Science and Research Branch, Islamic Azad University, Tehran, Iran. \\ ${ }^{5}$ Department of Environmental Science, Faculty of Environment and Energy, \\ Science and Research Branch, Islamic Azad University, Tehran, Iran.
}

http://dx.doi.org/10.12944/CWE.9.1.15

(Received: December 24, 2013; Accepted: February 15, 2014)

\begin{abstract}
Algae play an important role in all aquatic ecosystems by providing all living organisms of water bodies with preliminary nutrients and energy required. However, abnormal and excessive algal growth so-called algal bloom would be detrimental as much. Given the importance of algae in aquatic environment as well as their sensitivity to environmental changes, algal measurements are of key components of water quality monitoring programs. The algal blooms could include a variety of adverse impacts on environmental, social, cultural and economic environments. The present study is an overview on the algal growth, its mechanisms and mitigating strategies in aquatic ecosystems whereas in spite of the growing knowledge of human being of ecological, physiological, and functional conditions of eutrophication, a systematic understanding of algal blooms is still lacking.
\end{abstract}

Key words: Algal bloom, Phytoplankton, Aquatic ecosystems.

\section{INTRODUCTION}

All algae are not green and could be observed in a range of colors depending on the dominant pigment in their cells (Imamura et al. 2013). For example, if the chlorophyll a is dominant pigment, then the alga color will tend to green. Orange and red algae contain high level of carotene pigment. Micro-algae are in tow forms of Phytoplankton and Periphyton. Phytoplankton live in water column suspendedly while Periphyton survive through connection to the stones, sediment, stems of plants and aquatic organisms. Algae are single-celled observed individually or as a cluster (Clooney) or incandescent (filament). They belong to primary producers known as autotrophs.
Autotrophs, in the presence of sunlight, convert water and carbon dioxide into sugar (food). In this process, oxygen is produced as a byproduct which helps survival of fish and other aquatic organisms. Phytoplankton require to remain on water surface to absorb sunlight for photosynthesis. Increase in the number of algal cells is influenced by season, temperature, amount of sunlight penetrating the water column, the amount of inorganic nutrients (minerals) available to compete with other algal and aquatic plants and water retention time in lakes (Simpson,1991). For example, during the summer when light is available, the amount of phosphorus in the lake is controlled by the amount and abundance of algae. Thus, phosphorus has been considered as a limiting nutrient in most freshwater 
bodies. Excessive amount of algae known as algal bloom in the surface of lakes creates stinking and dense substrates. Algal blooming has become one of the key fields of study on eutrophication of water bodies in recent years (Wu and $\mathrm{Xu}, 2011)$. Due to the importance of algal bloom in aquatic ecosystems, the event mechanism has been studied by many researchers worldwide. Li Liu and Tang in 2012 reported spatial and temporal variations of algal bloom events in the coastal waters of the western South China Sea (SCS) from 1993 to 2007. They concluded that twenty-five algal bloom events occurred in summer in the coastal waters of South and Central Vietnam induced by wind-induced, coastal, nutrient upwelling and river discharges; a further eight events occurred in the coastal waters of North Vietnam. Yao et al. (2011)developed a directed Complex Networks (CNs) model of algal blooms based on the characteristics of CNs theory and the primary factors that influenced algal blooms.

$\mathrm{Ni}$ et al. (2010) assessed the impacts of algal blooms removal by chitosan-modified soils on zooplankton community in Taihu Lake, China. They found that Polymerase Chain ReactionDenatured Gradient Gel Electrophoresis (PCRDGGE) CR-DGGE could be applied to investigate the impacts of the environmental protection or restoration engineering on zooplankton community diversity. Barale et al. (2008) studied algal blooming patterns and anomalies in the Mediterranean Sea using the SeaWiFS data set (1998-2003). Asaeda et al. (2001) performed a numerical analysis to control algal blooms in reservoirs with a curtain.

The present study is an overview on the algal growth, its mechanisms and mitigating strategies in aquatic ecosystems whereas in spite of the growing knowledge of human being of ecological, physiological, and functional conditions of eutrophication, a systematic understanding of algal blooms is still lacking.

\section{Seasonal changes in algae}

Algae are very diverse group of organisms so that more than 40 species can coexist in a lake. However, the dominant algal species change throughout an annual cycle in lakes known as algal succession (Kortmann and Henry, 1990). Algal population is abundant in spring and early summer, when available light and nutrients are plenty and small numbers of organisms are feeding on algae. Coincides with the end of this phase, a Clear-Water Phase (CWP) phenomenon occurs in many lakes. Spring algal population is made up of small and edible species. At this stage, zooplankton dramatically increase and consume the algae, rapidly. As a result, water is very clear for several weeks. That is why the phenomenon is called "clear water phase". This algal population is gradually replaced by larger non-edible species in the form colonies which often covered by a gelatinous sheath. Since the concentration of available nutrients is limited in summer, the whole algal concentration in summer is lower than that of in spring (before the CWP). From the late summer and fall, the stored nutrients in the lake are mixed in the water column and a fresh supply of nutrients is produced. This let the algal population for seasonal re-blooming. During the winter months, algae are able to survive, but usually at low concentrations due to the lower amount of available sunlight and low water temperature (Green and Herron, 2001).

\section{Algal blooms}

Algae are useful and necessary for aquatic ecosystems and provide primary energy and nutrients for almost all living organisms. However, abnormally high levels of algal growth can cause interference in functionality of water bodies and reduce water aesthetics through declined water clarity. By shading, accumulated algae prevent light from reaching the roots of aquatic plants (macrophytes). Excessive algal growth increases dead algae resulting in their decomposition and decreasing DO in water bodies during summer. DO absence causes a condition known as Anoxia in which the fish are killed. High levels of algae may also increase the $\mathrm{pH}$ of the water bodies. Increased pH level seems to be a byproduct of increased photosynthesis of carbon dioxide. High pH levels are commonly seen in the late afternoon of sunny summers after the consumption of the $\mathrm{CO} 2$ by photosynthesis process. After sunset, the $\mathrm{pH}$ level may significantly be declined due to ending the photosynthesis process. These extreme fluctuations in $\mathrm{pH}$ can cause stress on sensitive aquatic species. There is also the concern that excessive amounts of algal material 
formed based on the reaction with chlorine used in water treatment, produce trihalomethanes as carcinogens. It is important to realize that the algal growth is occurred through natural cycles in natural ecosystems. The algal bloom is problematic as a result of direct human manipulation on the environment.Managers should be targeted towards maintaining health and natural algal levels in water bodies.

\section{Measuring algal concentrations}

Algal concentrations are measured to determine the eutrophication status in water bodies. Eutrophication is an indicator for the natural aging process of the lakes. Oligotrophic waters are waters with high clarity and depth as well as little algae contained. Water bodies with algal abundance, are eutrophic and often turbid. In the mid-process, medium-resolution lakes with medium algal levels are called mesotrophic. Simpson in 1991 declared that since algae are a strong indicator for environmental changes, in most of the monitoring programs, the algal concentrations are measured to determine changes in water quality.Algal and green plants require green pigments of chlorophyll a for photosynthesis. Considering that the ratio of chlorophyll a to biomass can vary among algal groups, the measurement of the chlorophyll a is considered as a reasonable estimate of algal concentrations. Chlorophyll $a$ is extracted with acetone. The concentrations are determined by spectrophotometer. This is probably the most reliable method for determining algal concentration whereas chlorophyll a is chemically extracted from algal cells. The advantages of this method are simplicity and stability sampling. There are some of the limitations associated with the measurement of algal biomass using this technique. As such, algae are not evenly distributed throughout the water bodies so it is necessary to take some water samples every day. In Vermont, the volunteers of the monitoring programs have addressed these limitations by taking an integrated sample proposed by EPA. In this method, the volunteers suggested double measurements of the Secchi depth and determined a water sample as the representative of the water column. Another limitation of this method is that the numbers of algal species have naturally a higher level of chlorophyll a than other algal species. In addition, the concentration of chlorophyll a s fluctuates during the day to maximize photosynthesis efficiency of algae. Constant and repeated measurements would be the best way to deal with these kinds of limitations. Taking water samples at the same time of day and the depth of the water column, the sample is collected, it can reduce these discrepancies. URIWW recommends that the samples of chlorophyll a should be taken between 10 am and 2 pm at the deepest point of the water body at a depth of $1 \mathrm{~m}$. One way for indirect measurement of concentration of chlorophyll a is to measure Secchi depth (estimation of waters clarity). The water clarity degree is a result of the amount of suspended maters in the water column. In areas with low sediment input, there is a strong correlation between concentration of chlorophyll a and Secchi depth. Besides, it is possible to estimate the potential algal content in water bodies using measuremnt of total phosphorus.

A high level of Chlorophyll a is approximately equal to $1-10 \mathrm{~g} / \mathrm{L} \mu$ for oligotrophic lakes and may reach up to $300 \mathrm{~g} / \mathrm{L} \mu$ in eutrophic lakes. In hypereutrophic lakes such as Hart Bees Poort in southern Africa, maximum Chlorophyll a could even reach $3000 \mathrm{~g} / \mathrm{L} \mu$.This dam has been eutrophicated as a result of high concentrations of phosphorus and nitrate on the River Crocodile, inflow and primary pollution source of domestic and industrial waste water (Minnesota Pollution Control Agency,2008). In overall, the eutrophication status of lakes is determined by chlorophyll a, total phosphorous and Secchi depth. Each of the parameters has its own weaknesses. Therefore, if these three parameters are considered together, they will contribute to present a complete picture of water quality and the relationship between water quality and algal growth in water bodies. By studying the algal species living in lakes, even more information can be obtained on water quality.

\section{Harmful Algal Blooms (HAB)}

The $\mathrm{HAB}$ is a kind of algal bloom imposing a negative impact on other organisms through releasing natural toxins and mechanical damage of other organisms often associated with large-scale marine mortality events. The HABs causes harmful effects to change marine mammals and sea turtles. In 2004, a volume of 107 dolphin deaths was occurred in Florida. Dangerous walls of North 
Atlantic were exposed to neurotoxin with high level of zooplankton pollution (Green et al. 2001).

\section{CONCLUSION}

Due to the growing concern about the nuisance algal growth in most lakes and water bodies it is of great importance to discover mechanisms for prevention, prediction and limitation of algal growth. As is apparent, the best way to restrict algal growth is to limit the amount of nutrients discharging into the lakes and water bodies. Chemicals, copper sulphate and organic synthesis can be added to waters bodies as pesticides for reducing algal growth. Aluminum buffer or calcium compounds bound with phosphate are sometimes added to in waters bodies make them unavailable to algae. When it is realized that these chemicals are effective in reducing algal growth, the use of these materials requires permission from the environmental authorized origination and must be done by an authorized user. Other control strategies are artificial aeration, biological control and physical elimination of algae. In aeration mechanism, oxygen is added to water bodies in order to inactive phosphorus or to reduce the effects of algal bloom. Of biological controlling methods could be pointed out algal feeders which can restrict number of algae in water bodies. Physical removal of algae could be water filtering from algae. This controlling method has different outcomes and may also be costly. The best controlling method is to limit nutrients in water bodies before getting increased. Until algal levels are not annoying, they play an indispensable role in healthy ecosystems.

\section{REFERENCES}

1. Li Liu Ch., Tang D. Spatial and temporal variations in algal blooms in the coastal waters of the western South China Sea. Journal of Hydro-environment Research, 6(3), 239-247 (2012).

2. Yao J., Xiao P., Zhang Y., Zhan M., Cheng J. A mathematical model of algal blooms based on the characteristics of complex networks theory. Ecological Modelling, 222(20-22), 3727-3733 (2011).

3. Assmy P., Smetacek V. Algal Blooms. Encyclopedia of Microbiology (Third Edition), 27-41 (2009).

4. Ni J., Yu Y., Feng W., Yan Q., Pan G., Yang B., Zhang X., Li X. Impacts of algal blooms removal by chitosan-modified soils on zooplankton community in Taihu Lake, China. Journal of Environmental Sciences, 22(10), 1500-1507 (2010).

5. Wu G., Xu Z. Prediction of algal blooming using EFDC model: Case study in the
Daoxiang Lake. Ecological Modelling, 222(6), 1245-1252 (2011).

6. Barale V., Jaquet J.-M., Ndiaye M. Algal blooming patterns and anomalies in the Mediterranean Sea as derived from the SeaWiFS data set (1998-2003). Remote Sensing of Environment, 112(8), 3300-3313 (2008).

7. Asaeda T., Pham H.S., NimalPriyantha D.G., Manatunge J., Hocking G.C. Control of algal blooms in reservoirs with a curtain: a numerical analysis. Ecological Engineering, 16(3), 395-404 (2001).

8. Imamura S., Ishiwata A., Watanabe S., Yoshikawa H., Tanaka K. Expression of budding yeast FKBP12 confers rapamycin susceptibility to the unicellular red alga Cyanidioschyzonmerolae. Biochemical and Biophysical Research Communications, (2), 264-269 (2013). 